\title{
5.36 Gbit/s OFDM optical wireless communication link over the underwater channel
}

\author{
João H. Araújo ${ }^{1,2}$, Rafael Kraemer ${ }^{1}$, Joana S. Tavares ${ }^{1,3}$, Francisco Pereira ${ }^{2}$, H. M. Salgado ${ }^{1,3}$, and L. M. Pessoa ${ }^{1,3}$ \\ ${ }^{1}$ INESC TEC - Institute for Systems and Computer Engineering, Technology and Science, Porto, Portugal \\ ${ }^{2}$ School of Engineering, Polytechnic Institute of Porto, Porto, Portugal \\ ${ }^{3}$ Faculty of Engineering, University of Porto, Porto, Portugal \\ e-mail: \{joao.h.araujo, rafael.kraemer, joana.s.tavares, henrique.salgado, luis.m.pessoa\}@inesctec.pt, fdp@isep.ipp.pt
}

\begin{abstract}
An OFDM transmission system is reported based on a directly modulated blue LASER diode, for high bit rate underwater optical communication applications. The 256 subcarriers 16-QAM signal is transmitted over a total distance of $2.4 \mathrm{~m}$ underwater with an EVM lower than $-28.5 \mathrm{~dB}$ for a $250 \mathrm{MHz}$ bandwidth and $-16.5 \mathrm{~dB}$ for a $2 \mathrm{GHz}$ bandwidth, the BER being lower than the forward error corrector limit. At the maximum bandwidth of $2 \mathrm{GHz}$ a transmission rate of $5.36 \mathrm{Gbit} / \mathrm{s}$ is achieved.
\end{abstract}

Index Terms-Underwater optical wireless communications, high-speed optical communications system, 16-QAM/OFDM.

\section{INTRODUCTION}

$\mathbf{T}$ HE exploitation of the ocean natural resources has recently been the subject of growing interest. The sustainable management of these marine resources calls for an urgent exploration of the seas to effectively characterize, protect, and preserve marine life and biodiversity. In this scope, unmanned underwater vehicles are an important asset that can be used to monitor and control the oceans in applications such as marine fauna investigation, environmental monitoring, off-shore oil and gas extraction industry control, and national surveillance [1]. In these scenarios, large amounts of data may be required to be collected from underwater sensors and vehicles. Hence, the research of new wireless communication systems and techniques plays an important role in the exploration of the ocean, ensuring that data transmission is increasingly optimized to the modern requirements [2].

Acoustic communications are the most common communication model in the underwater environment. However, they are severely affected by the low bandwidth, latency, and propagation speed of the acoustic waves and by the existence of multipath propagation and Doppler effect. Radiofrequency (RF) communications are limited by the attenuation caused by the high conductivity and permittivity of the saline water and the low bandwidth due to the scarcity of the RF spectrum [3]. None of the two referred models are suitable for the growing data rate requirements.

This work is financed by the ERDF - European Regional Development Fund through the Operational Programme for Competitiveness and Internationalisation - COMPETE 2020 Programme and by National Funds through the Portuguese funding agency, FCT - Fundação para a Ciência e a Tecnologia within project POCI-01-0145-FEDER-031971.
On the other hand, optical communications have gathered considerable interest in academic, military, and industrial context with the efficient development of high bandwidth and robustness, low cost, and low latency short-range communication systems. Underwater optical wireless communications (UOWC) are very challenging since optical signals are affected by the misalignment (between the transmitter and receiver) due to the ocean turbulence and by the attenuation existing in the underwater communication channel [2]. In spite of the low loss at the blue-green region of the electromagnetic spectrum, optical waves are also affected by scattering due to the presence of suspended particles, especially in turbid waters. All of the referred parameters contribute strongly to a degradation of the error vector magnitude (EVM) and consequently the bit error rate (BER) performance. Still, they are an attractive solution for mid-range $(\sim 100 \mathrm{~m})$ and high-speed transmission rates, suitable for unmanned vehicles mission scenarios.

UOWC systems can be designed using either light-emitting diodes (LEDs) or LASER diodes (LDs) as optical sources at the transmitter. In spite of the lower cost, longer lifetime, and reliability LEDs inherently have higher divergence being limited in terms of modulation bandwidth. Previous studies have shown that the use of directly modulated LDs are an attractive option to increase the available modulation bandwidth with respect to LED-based systems which are limited to hundreds of Mbit/s [4].

Moreover, orthogonal frequency division multiplexing (OFDM) offers many advantages in UOWC systems for being fairly robust, allowing to increase the received signal quality, considering that the underwater channel severely degrades the optical signal. This technique comprises a cyclic prefix (CP), which consists of copying the last samples of an OFDM symbol to the beginning of each symbol, allowing to reduce the multipath effect and, consequently, the intersymbol interference (ISI). In [5], a blue LD is used for the transmission of an OFDM/16-QAM signal over a distance of $6.8 \mathrm{~m}$ at a rate of $7.2 \mathrm{Gbit} / \mathrm{s}$ with a received EVM of $16.4 \%(-15.2 \mathrm{~dB})$. Here, we report the design and characterization of an OFDM wireless communication link over the underwater channel with an EVM corresponding to a BER lower than the required forward error corrector limit of $3.8 \times 10^{-3}$, for all the considered OFDM signal bandwidth. 


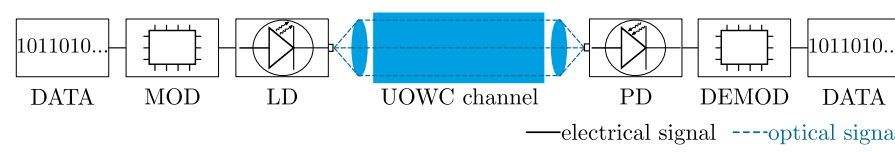

Fig. 1. Block diagram of a high-speed UOWC system.

The remainder of this paper is organized as follows: in Section II we first describe the UOWC system, where the preliminary details of some elements that constitute it are referred to; in Section III we present the experimental setup whereupon all the elements used are described in detail, also presenting the overall system architecture; in Section IV we show and compare the experimental results of the UOWC system through the analysis of EVM and the constellation maps. The final conclusions are presented in Section V.

\section{UNDERWATER COMMUNICATION SYSTEM}

The necessary elements for the establishment of a UOWC are depicted in Fig. 1.

Firstly, the data to be transmitted was conveniently generated in MATLAB from a pseudorandom binary sequence (PRBS) and modulated by 256 OFDM subcarriers, employing DCbiased Optical OFDM (DCO-OFDM) scheme. Therefore, the data are mapped in 16-QAM symbols and converted to OFDM signals (in the time domain) through the IFFT technique, and the $\mathrm{CP}$ is added to the beginning of each symbol. To mitigate the channel-induced ISI, it was used a CP with a length of $1 / 8$ from the total number of subcarriers. For channel estimation and carrier-phase tracking on the receiver, each OFDM symbol contained 8 pilots and for signal synchronization in the receiver and ease of implementation of eventual filters, 55 subcarriers (28 to left and 27 to the right) were used as guard bands. We considered for the modulated OFDM signals the following bandwidth (BW): 250, 500, 750, 1000, 1200, 1500, and $2000 \mathrm{MHz}$, whose spectra are represented in Fig. 2. For comparison, the central frequency was set to $800 \mathrm{MHz}$ for all cases.

A blue $\operatorname{LD}\left(\lambda_{p}=450 \mathrm{~nm}\right)$ with a spectral width of $2 \mathrm{~nm}$ and an optical output power of $80 \mathrm{~mW}$ at an operating temperature of $25^{\circ} \mathrm{C}$ is used to perform the electrical-to-optical conversion in order to ensure a high-speed transmission.

The signal is delivered to the underwater channel by an optical lens and is propagated across the communication channel until it is collected by another optical lens. The optical-toelectrical conversion is accomplished by a high-speed Si PIN photodiode (PD) with an operating bandwidth of $7.45 \mathrm{GHz}$ and then the electrical signal is ready to be demodulated. After the $\mathrm{CP}$ is removed, the FFT technique is applied to the signal and, after demapping the 16-QAM symbols, the original information is retrieved by the receiver.

\section{EXPERIMENTAL SETUP}

Fig. 3 shows the UOWC experimental setup architecture. Firstly, the MATLAB-generated OFDM signal is sent to a $12 \mathrm{GSa} / \mathrm{s}$ 12-bit arbitrary waveform generator (AWG), that

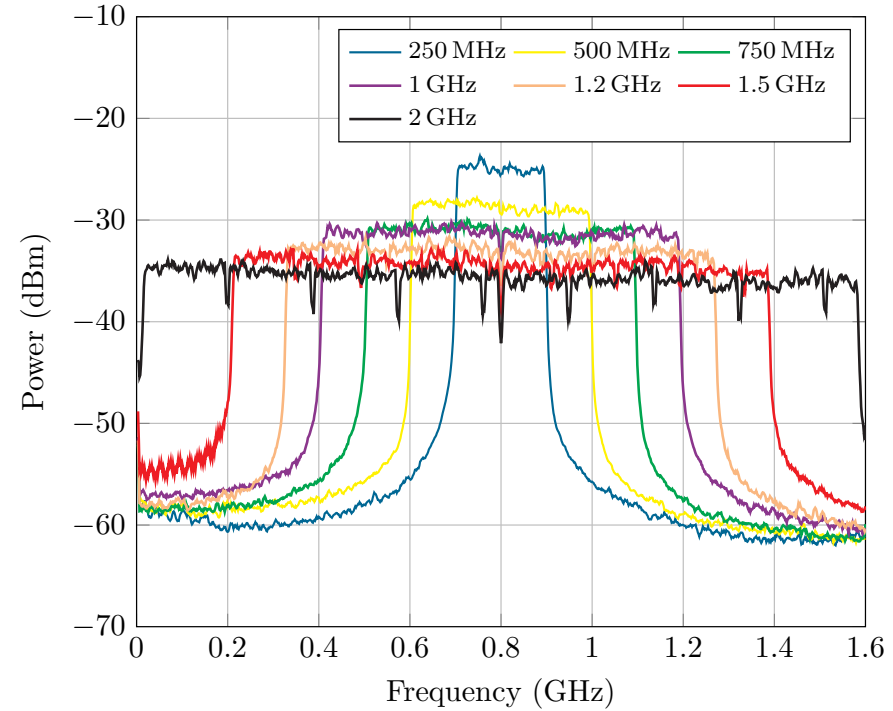

Fig. 2. OFDM spectra for different signal bandwidth at $800 \mathrm{MHz}$ with a resolution bandwidth of $1 \mathrm{MHz}$.

realizes the digital-to-analog conversion of the signal, and delivers it to the homemade transmitter module printed circuit board (PCB). This PCB includes a current source for biasing the LD, a bias-tee, and a temperature control circuit based on a thermoelectric cooler (TEC) for establishing the LD temperature at $\sim 25^{\circ} \mathrm{C}$. At the output of the bias-tee, both RF signal and bias are combined which directly modulates the LD. Prior to the modulation, an additional circuit is used to match the impedance of the LD [6].

The optical signal is converted into a parallel beam by the plano-convex lens (Thorlabs LA1951-ML, focal length of $25.4 \mathrm{~mm}$, depicted in Fig. 4a) and propagated $30 \mathrm{~cm}$ in freespace until it reaches the glass water tank $(1.20 \mathrm{~m} \times 0.50 \mathrm{~m} \times$ $0.50 \mathrm{~m}$ ) filled with fresh tap water. The optical OFDM signal propagates across the underwater channel for the total length of the tank, i.e., $1.20 \mathrm{~m}$. The optical signal leaves the water tank and propagates an additional $16 \mathrm{~cm}$ in free-space until it is reflected by a broadband dielectric mirror (Thorlabs BBSQ1E02, illustrated in Fig. 4c) whose average reflectance is higher than $99 \%$. Afterward, the signal propagates back through $16 \mathrm{~cm}$ in free-space until it enters again and propagates $1.20 \mathrm{~m}$ more in the water tank. Finally, the optical signal leaves the water tank and further propagates $23 \mathrm{~cm}$ in free-space until it is finally collected by the convex-plano lens (shown in Fig. 4b) and fed to the PD followed by a preamplifier and a bias-tee to remove the DC component.

The amplified electrical signal is captured by a digital storage oscilloscope (DSO) that collects (time-domain) samples and sends them to the computer program 89600 VSA that accomplishes the demodulation process and evaluates the transmission performance, showing the OFDM signal spectrum and received constellation map, among other parameters.

The setup assembled in the laboratory is depicted in Fig. 5, where one can observe the AWG, the transmitter module, the 


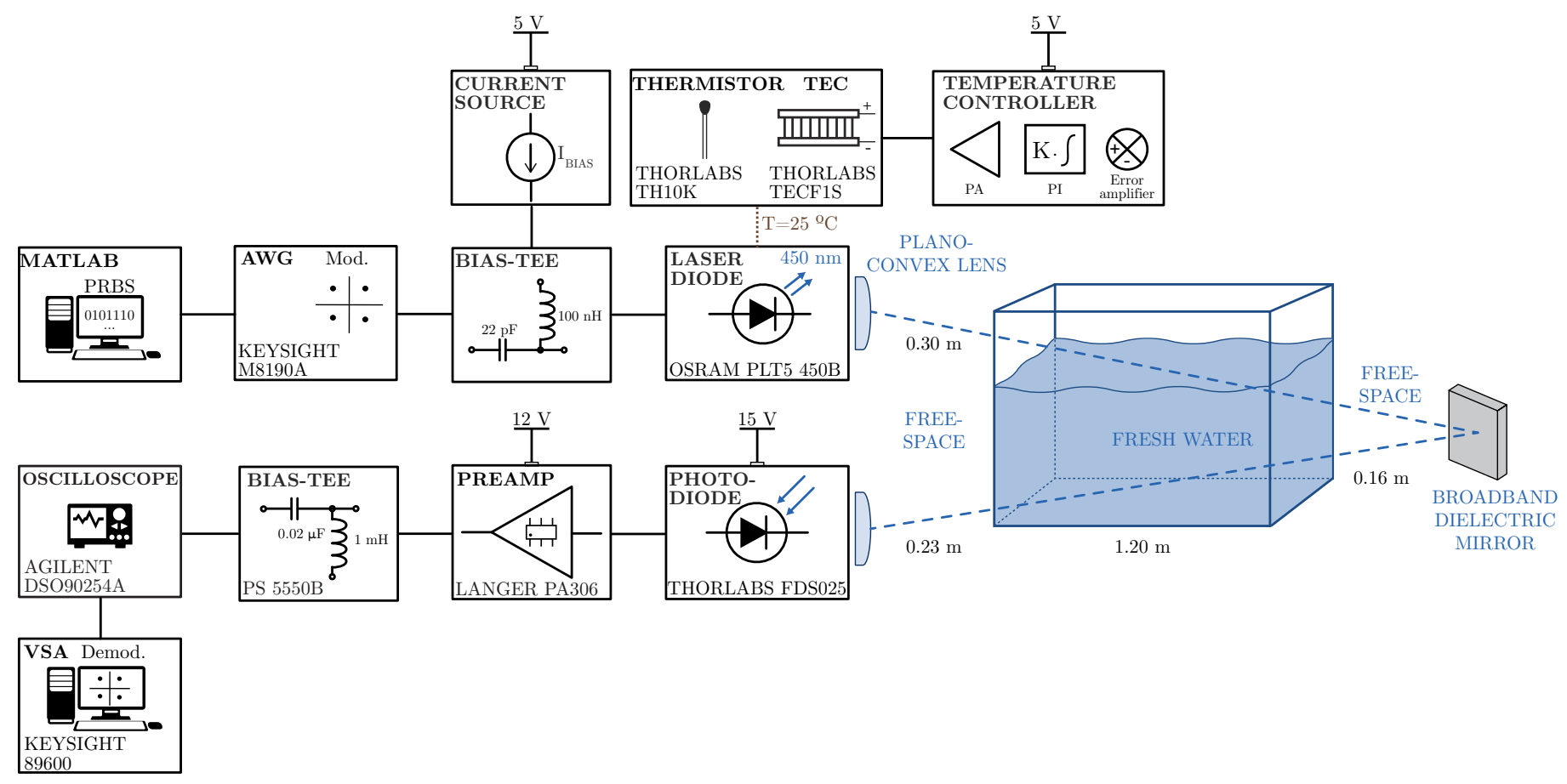

Fig. 3. Experimental setup architecture for a $3.25 \mathrm{~m}$ UOWC.

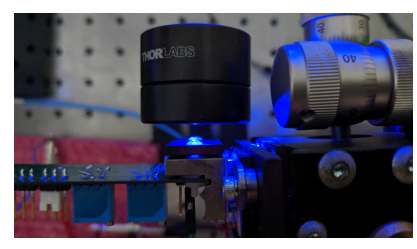

(a) LD and plano-convex lens.

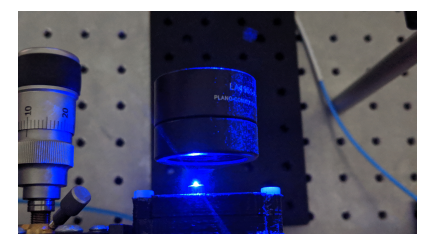

(b) PD and convex-plano lens.

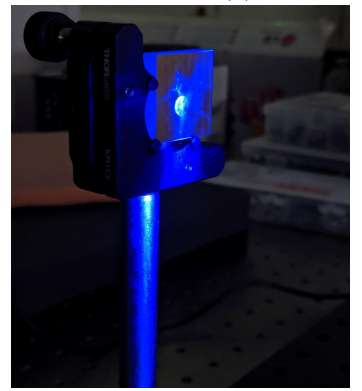

(c) Reflective mirror.

Fig. 4. Optical devices inherent to the UOWC system.

lenses, the water tank, the multiple reflections of the optical signal, the receiver module, the DSO, and the demodulation results in the computer.

\section{EXPERIMENTAL RESUlts}

To better understand the proposed system behavior, the frequency response of the blue $\mathrm{LD}$, air+water communication channel, and the PD set was evaluated for different LD bias currents, illustrated in Fig. 6. Increasing the LD bias current produced a decreased magnitude of the resonance frequency of the LD which also shifts to larger frequencies. Operating the

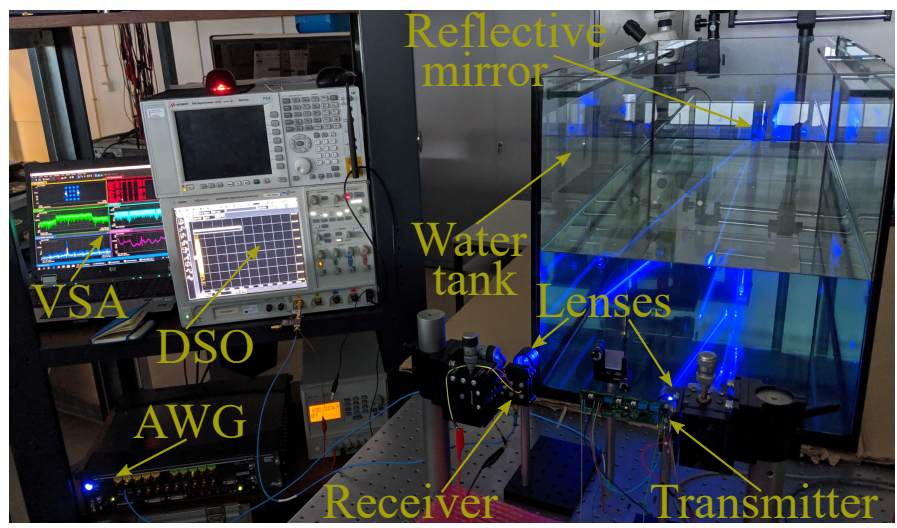

Fig. 5. Experimental laboratory setup for a $3.25 \mathrm{~m}$ UOWC.

LD at low bias currents $(30 \mathrm{~mA})$ in this case has the advantage of compensating the fast roll off of the transfer function which is attributed to the LD packaging parasitics. Moreover, all bias currents show a considerable dip at around $2 \mathrm{GHz}$. Given the measured frequency response of the system, the UOWC was considered to operate in the range of $100 \mathrm{MHz}$ and $2 \mathrm{GHz}$, more specifically the spectra represented in Fig. 2.

For this kind of OFDM-based communication systems, the typical metric used to evaluate its performance is the EVM, which quantifies the residual noise and distortion of the transmitted signal, i.e., it corresponds to the scalar distance between the two I vs. Q plane phasors of the received signal constellation map and the reference (ideal) corresponding signal, regarding the considered modulation scheme. 


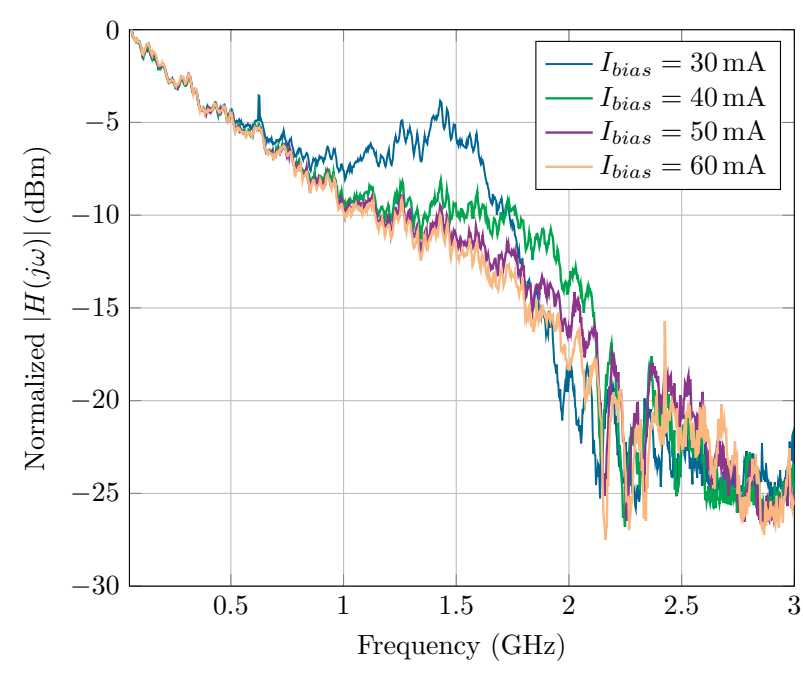

Fig. 6. UOWC system frequency response for different LD bias currents.

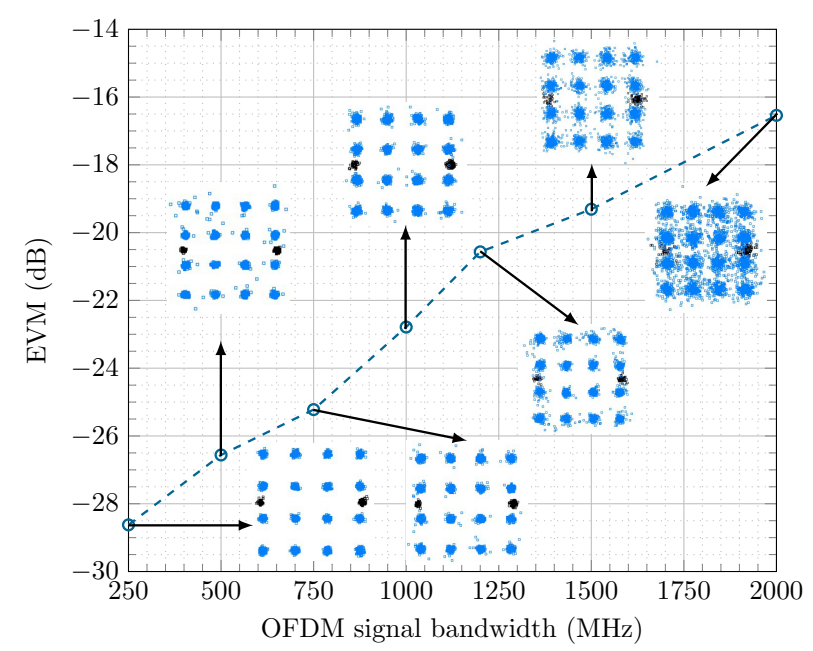

Fig. 7. UOWC system EVM for different OFDM signal bandwidth and corresponding constellation maps with $30 \mathrm{~mA}$ of LD bias current.

After demodulating the signal, the constellation maps and the EVM were obtained. The measured EVM for different OFDM signal bandwidth and corresponding transmission rate is described in Table I and the characteristic of the EVM versus the OFDM signal bandwidth are illustrated in Fig. 7.

The results show that the EVM increases with the increasing OFDM signal bandwidth, as expected. Clear constellation maps could be obtained up to $1500 \mathrm{MHz}$ and from that frequency, we observe a severe degradation, especially at $2000 \mathrm{MHz}$, due to the bandwidth limitation of the system. Increasing the bandwidth from $250 \mathrm{MHz}$ to $2000 \mathrm{MHz}$ enlarges the LD transmission capacity from $0.67 \mathrm{Gbit} / \mathrm{s}$ to $5.36 \mathrm{Gbit} / \mathrm{s}$ at the expense of degraded BER up to $1 \times 10^{-3}$, although less than the FEC limit $\left(3.8 \times 10^{-3}\right)$ [7], [8].
TABLE I

MEASURED EVM FOR DIFFERENT OFDM SIGNAL BANDWIDTH AND CORRESPONDING TRANSMISSION RATES.

\begin{tabular}{ccc}
\hline OFDM Bandwidth $(\mathrm{MHz})$ & Transmission Rate (Gbit/s) & EVM (dB) \\
\hline 250 & 0.67 & -28.62 \\
\hline 500 & 1.34 & -26.56 \\
\hline 750 & 2.01 & -25.22 \\
\hline 1000 & 2.68 & -22.79 \\
\hline 1200 & 3.22 & -20.57 \\
\hline 1500 & 4.02 & -19.31 \\
\hline 2000 & 5.36 & -16.54
\end{tabular}

\section{CONCLUSiON}

In this paper, an underwater OFDM transmission is performed based on a directly modulated blue LD. The OFDM transmitter operating over the range of $100 \mathrm{MHz}$ to $2000 \mathrm{MHz}$, with 256 subcarriers and 16-QAM subcarrier modulation, can achieve a total transmission rate of $5.36 \mathrm{Gbit} / \mathrm{s}$ over a $2.4 \mathrm{~m}$ of the underwater channel.

\section{REFERENCES}

[1] K. Awan, D. P. A. Shah, K. Iqbal, S. Gillani, W. Ahmad, and Y. Nam, "Underwater wireless sensor networks: A review of recent issues and challenges," Wireless Communications and Mobile Computing, vol. 2019, pp. 1-20, 012019.

[2] X. Sun, C. H. Kang, M. Kong, O. Alkhazragi, Y. Guo, M. Ouhssain, Y. Weng, B. H. Jones, T. K. Ng, and B. S. Ooi, "A review on practical considerations and solutions in underwater wireless optical communication," Journal of Lightwave Technology, vol. 38, no. 2, pp. 421-431, Jan 2020.

[3] C. Gussen, P. Diniz, M. Campos, W. Martins, F. Costa, and J. Gois, "A survey of underwater wireless communication technologies," Journal of Communication and Information Systems, vol. 31, no. 1, Oct. 2016.

[4] J. Xu, M. Kong, A. Lin, Y. Song, X. Yu, F. Qu, J. Han, and N. Deng, "OFDM-based broadband underwater wireless optical communication system using a compact blue LED," Optics Communications, vol. 369, pp. $100-105,2016$.

[5] T.-C. Wu, Y.-C. Chi, H.-Y. Wang, C.-T. Tsai, and G.-R. Lin, "Blue laser diode enables underwater communication at $12.4 \mathrm{Gbps}$," Scientific Reports, vol. 7, no. 1, jan. 2017

[6] J. H. Araújo, R. Kraemer, H. M. Santos, F. Pereira, H. M. Salgado, and L. M. Pessoa, "LASER Diode-Based transmitter module for optical wireless communications," in 2020 12th International Symposium on Communication Systems, Networks and Digital Signal Processing (CSNDSP) (CSNDSP2020), Porto, Portugal, Jul. 2020.

[7] R. A. Shafik, M. S. Rahman, and A. R. Islam, "On the extended relationships among EVM, BER and SNR as performance metrics," in 2006 International Conference on Electrical and Computer Engineering, Dec 2006, pp. 408-411.

[8] J. Wang, C. Lu, S. Li, and Z. Xu, “100 m/500 Mbps underwater optical wireless communication using an NRZ-OOK modulated $520 \mathrm{~nm}$ laser diode," Opt. Express, vol. 27, no. 9, pp. 12 171-12 181, Apr 2019. 